\title{
In Silico Screening of Mutated K-Ras Inhibitors from Malaysian Typhonium flagelliforme for Non-Small Cell Lung Cancer
}

\author{
Ayesha Fatima and H. F. Yee \\ Department of Pharmaceutical Technology, Faculty of Pharmaceutical Sciences, UCSI University, Jalan Menara Gading 1, \\ Taman Connaught, Cheras, 56000 Kuala Lumpur, Malaysia
}

Correspondence should be addressed to Ayesha Fatima; ayesha@ucsiuniversity.edu.my

Received 6 February 2014; Accepted 19 August 2014; Published 21 September 2014

Academic Editor: Tatsuya Akutsu

Copyright (C) 2014 A. Fatima and H. F. Yee. This is an open access article distributed under the Creative Commons Attribution License, which permits unrestricted use, distribution, and reproduction in any medium, provided the original work is properly cited.

K-ras is an oncogenic GTPase responsible for at least $15-25 \%$ of all non-small cell lung cancer cases worldwide. Lung cancer of both types is increasing with an alarming rate due to smoking habits in Malaysia among men and women. Natural products always offer alternate treatment therapies that are safe and effective. Typhonium flagelliforme or Keladi Tikus is a local plant known to possess anticancer properties. The whole extract is considered more potent than individual constituents. Since K-ras is the key protein in lung cancer, our aim was to identify the constituents of the plant that could target the mutated K-ras. Using docking strategies, reported potentially active compounds of Typhonium flagelliforme were docked into the allosteric surface pockets and switch regions of the K-ras protein to identify possible inhibitors. The selected ligands were found to have a high binding affinity for the switch II and the interphase region of the ras-SOS binding surface.

\section{Introduction}

Cancer is a major health problem in Malaysia with a total of 2,048 cases registered with National Cancer Registry (NCR) in 2006 [1]. The disease is now the third leading cause of premature deaths in our country. Lung cancer is among the top five cancers affecting both male and female in Malaysia at 9.4 percent in peninsula Malaysia with 2100 Malaysians diagnosed every year [2,3]. Smoking related diseases are increasing in Malaysia especially lung cancer [3, 4]. A recent study conducted by Liam et al. (2013) highlighted adenocarcinomas as the most frequent types of cancer among Malaysian men and women, smoker, and nonsmokers. With an incidence rate of 109.8 cases of cancer per 100,000 , it is imperative to find treatments that are safe and effective [5].

Lung cancer can be divided into two major classes based on its biology, therapy, and prognosis, namely, non-small cell lung cancer (NSCLC) and small cell lung cancer (SCLC). NSCLC accounts for $75 \%$ to $80 \%$ of all lung cancer incidents while small cell lung cancer accounts for $15 \%$ to $25 \%$ of all lung cancer [6].

The ras pathway is an important signaling pathway that allows cell proliferation in response to stimulation of the epidermal growth factor receptor $[7,8]$. These signals affect the production and regulation of other key proteins involved in cell proliferation. Studies have reported that K-ras mutation occurring in NSCLC varies between $16 \%$ and $40 \%$ [8]. K-ras, a GTPase, also known as V-Ki-ras-2 (Kirsten rat sarcoma viral oncogene) is a protein that in human is encoded by the K-Ras gene [9]. The normal protein is an essential part of the ras signaling pathway acting as a molecular switch. In the "off" state, it is bound to the guanine diphosphate nucleotide (GDP). It is turned on via the growth factor stimuli. The guanine nucleotide exchange factor, also known as the son of sevenless (SOS) protein, and the growth factor receptor-bound protein 2 (Grb-2) together enable the K-ras to become activated by exchanging the GDP molecule for the more active guanine triphosphate nucleotide (GTP). Once turned "on," it recruits and activates downstream proteins 
necessary for the propagation of growth factor and other receptor signals. It possesses an intrinsic weak enzymatic activity which is enhanced by interaction with the GTPase activating protein (GAP) leading to cleavage of the terminal phosphate of the nucleotide guanine triphosphate (GTP) converting it into the guanine diphosphate (GDP). Upon conversion of GTP to GDP, K-ras is switched "off." Despite being a weak GTPase, K-ras possesses two very important features involved in its switching "on" and "off." Called switches because of their ability to change the conformation of the protein in the active and inactive state, the regions are involved in interacting with the nucleotides. The guanine nucleotide pocket of the K-ras is highly conserved and is lined with residues 11-16 [10,11]. Because of the specific interactions of amino acid residues of this region with the GTP, mutations at the 12 and 13 amino acid positions in the enzyme lead to permanent cell proliferation because it cannot be hydrolysed and hence, the ras signaling function is unable to be turned "off" [12-14]. The mutated K-ras (mut-Kras) is an interesting drug target of several studies [15-18]. The major reason being that it provides fast resistance to the available drug therapy. Several EGFR, MEK inhibitors have been tried in single and combination. However, drug resistance develops quickly $[17,19-21]$.

Medicinal plants with anti-cancer effects are commonly used as alternative medicine because of their safety and toxicity profiles. Several herbal medicines have been studied for finding effective treatment of lung cancer. Only few studies on the use of Malaysian medicinal plants as treatment options have been reported $[4,21,22]$. Typhonium flagelliforme (Keladi tikus) is one such plant that is found locally in Malaysia that has been studied for its inhibition of proliferation in human lung cancer cell line. Its active ingredients including phytol and its derivative, hexadecanoic acid, 1-hexadecene, and pheophorbide related compoundshave shown some promising results as anticancer when whole extracts have been used. Lai et al. reported that they could not find single constituents as effective when compared to the extract $[22,23]$.

Computer studies have recently provided insights into the mechanics of K-ras protein [24-26]. Researchers have offered in depth study of the various mutations and the effect they have on the "on-off" states of the protein. With sophisticated software being available to researchers, they have recently reported direct inhibition of the protein as therapeutic target. Maurer et al. (2012) have carried out an in-depth study of the allosteric binding pockets on the protein that maybe targeted in the "off" state of the mut-K-ras [24].

In this study we explored the inhibitory effects of the some of the reported potent constituents of T. flagelliforme on the lung cancer cell lines using docking studies with Autodock Vina [27]. We used the reported structures of the active ingredients and docked them into reported allosteric binding sites $[25,28]$ on the mut-K-ras to determine the probable binding sites of the constituents. We also tried to relate the experimental results obtained by Lai et al. [22, 23] to our computational observations to gain meaningful insights into the use of the proposed plant constituents as probable inhibitors.

\section{Materials and Methods}

The three dimensional structures of the G12D mutated K-ras structure inhibitor bound to SOS pocket (PDB ID: 4DST), and two G12C mutated K-ras structure bound to allosteric sites (PDB ID: 4LUC and 4LYF). Autodock Vina 4.2 [27] was used to dock all ligands to the K-ras protein. Before that, we used Autodock tools downloaded from The Scripps Research Institute to prepare the ligand and protein file [29, 30]. All water molecules were removed and Kollman charges added as described in the Autodock Vina 4.2 manual [31, 32]. The grid box dimensions were obtained from the grid box widget by keeping the bound ligand sites as box centers. Control studies were performed with all ligand bound in the crystal structures before docking with test ligands from $T$. flagelliforme. Pheophorbide a and two related epimers were drawn in ChemSketch [33] based on reported structure by Lai et al. [22] while hexadecene and hexadecanoic acid structure were obtained from Pubchem. The 2D structures of the active constituents are tabulated in Table 1.

\section{Results and Discussions}

The results of highest binding affinity of the five active constituents for the GTP bound K-ras as well as the mutated protein structures are tabulated in Table 2.

T. flagelliforme is a local Malaysian plant with anticancer activity when taken as a fresh juice prepared from freshly crushed plant. Researchers have reported its activity in lung cancer as a whole extract in dichloromethane [22, 23]. Lai et al. (2010) reported that the extract contained at least 11 chemical compounds of hydrophobic character [22]. The most predominant compounds were pherophorbide a, pheophorbide a', pyropheophorbide a, methyl pyropheophorbide a, hexadecanoic acid, oleic acid, linoleic acid, linolenic acid, campesterol, stigmasterol, and $\beta$-sitosterol. The in vitro studies conducted by the group on the NCI-H23 lung cancer cell line had concluded that individual compound isolates had failed to show a significant anti-cancer behavior. However, the whole extracts were found to have an $\mathrm{IC}_{50}$ of $2.7 \mu \mathrm{g} / \mathrm{mL}$ suggesting that components had a synergistic effect on antiproliferation of cancerous cells. They also suggested that the constituents showed increased activity upon exposure to light. The authors had also earlier reported an active whole extract of the plant comprising of hexadecanoic acid, 1-hexadecene, phytol, and a phytol derivative with an $\mathrm{IC}_{50}$ of $7.5 \mu \mathrm{g} / \mathrm{mL}$ against NCI-H23 cell lines [23].

Mohan et al. (2010) conducted their study on the leukemic cells and showed the selectivity of the dichloromethane fractions for the cancerous cells. However, they did not indicate the effect of any particular constituent on the cancer cells [34].

In our study we focused on determining whether the proposed constituents by Lai et al. $(2008,2010)$ were able to target the K-ras protein either directly or allosterically. For direct inhibition we docked the selected ligands at or near the SOS binding pocket using the PDB structure 4DST [24]. The target binding site proposed by the authors is near the ras switches I and II and binding to this area was indicated 
TABLE 1: Two dimensional structures of the constituents used in the study.<smiles>C=CCCCC=CCCCCC</smiles>

Pheophorbide a epimer
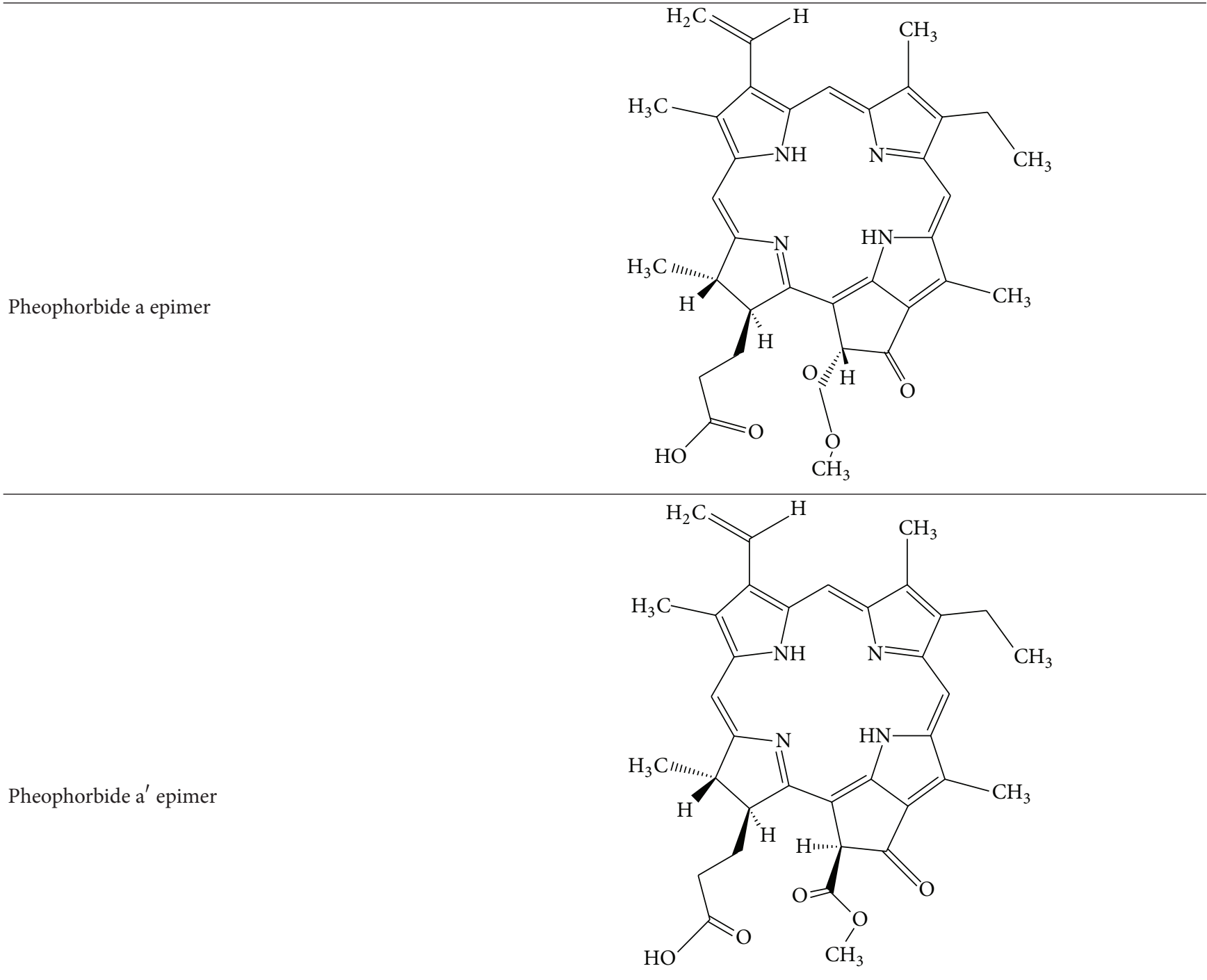
TABle 1: Continued.

Name

Structure

Pyropheophorbide epimer

TABLE 2: Binding affinity values obtained for the control and test ligands.

\begin{tabular}{|c|c|c|c|c|c|c|}
\hline \multirow{2}{*}{ Protein } & \multicolumn{6}{|c|}{ Binding affinity $\mathrm{Kcal} / \mathrm{mol}$} \\
\hline & Control & Pheophorbide A & Pheophorbide $\mathrm{A}^{\prime}$ & Pyropheophorbide A & 1-Hexadecene & Hexadecanoic acid \\
\hline $\begin{array}{l}\text { G12D mutated } \\
\text { K-ras GDP SOS } \\
\text { binding pocket } \\
\text { (4DST) }\end{array}$ & -5.4 & -7.2 & -7.5 & -7.1 & -4.0 & -4.1 \\
\hline $\begin{array}{l}\text { G12C mutated } \\
\text { K-ras GDP } \\
\text { allosteric binding } \\
\text { pocket ( } 4 \text { LUC) }\end{array}$ & -8.0 & -7.0 & -6.8 & -7.3 & -4.8 & -4.6 \\
\hline $\begin{array}{l}\text { G12C mutated } \\
\text { K-ras GDP } \\
\text { allosteric binding } \\
\text { pocket ( } 4 \mathrm{LYF} \text { ) }\end{array}$ & -6.7 & -6.7 & -6.6 & -6.5 & -4.5 & -5.0 \\
\hline
\end{tabular}

as interfering with ras-SOS binding surface [24]. This finding is important because the Ras-SOS complex is essential for activation of the K-ras since SOS initiates the GTP exchange process to the protein. According to the resolved structure of the ras-SOS complex (PDB ID: 1BKD) [35], the CDC25 binding region is tightly bound to the Switch II of ras and causes the disruption of the GDP bound structure [35]. Tyr 64 of the ras appears to be the anchoring residue for the Ras-SOS complex. Hence, the inhibitors should be designed to target the switch regions or the binding surface between the proteinprotein complex. Where small molecules can modulate the switch regions of the ras due to space confinement, larger molecules can target the accessible surface areas between the protein-protein complex.

Our semiflexible docking experiment on the K-ras molecule with control ligand 4,6-dichloro-2-methyl-3-aminoethylindole (4DST) [24] had a binding affinity of $-5.4 \mathrm{Kcal} / \mathrm{mol}$. Among the test ligands the highest binding affinities were shown by the pheophorbide epimers. The observed docked

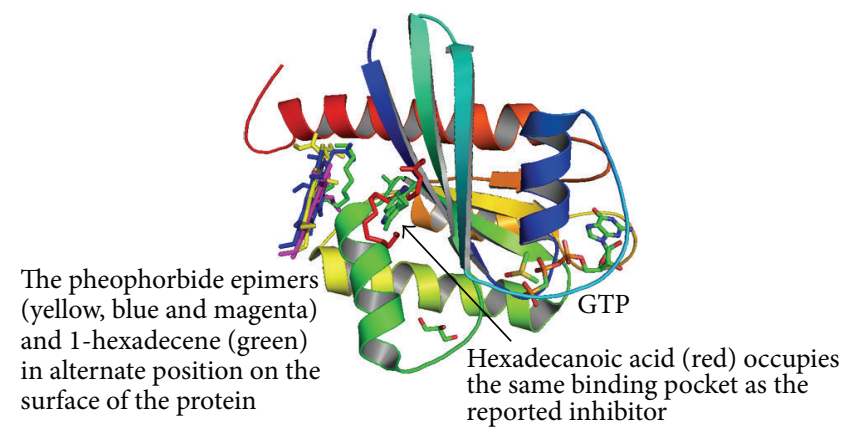

FIGURE 1: Docked poses of flagelliforme constituents to GTP bound K-ras.

poses, given in Figure 1, showed that the epimers were not near the binding site of the control ligand.

The test ligand that showed affinity for the same binding site as the control molecule was hexadecanoic acid with 
binding affinity $-4.1 \mathrm{Kcal} / \mathrm{mol}$. It appeared to overlap the control molecule at the same position. The authors suggested that the binding pocket involves residues Lys5, Leu6, Val7, Ile55, Leu56, and Thr74 [24]. Our results showed mostly the same binding site except that Thr 74 was placed a bit further from the ligands but within $5 \AA$. Our results showed the binding pocket residues to be Lys5, Leu6, Val7, Glu37, Ser39, Arg40, Asp54, Ile55, Leu56, Gln70, Tyr71, Met72, Thr74, and Gly75. They further elaborated that the binding of the indole derivative expanded the pocket to accommodate the ligand. A recent report by Grant et al. (2011) also established this region as one of the allosteric binding pockets important in finding inhibitors for K-ras [25]. Hexadecanoic acid is a long chained hydrocarbon that folds into a U-shape when docking into the pocket. This folding makes the molecule snugly fit into the pocket. From this result we can assume that when the cancer cells were incubated with the T. flagelliforme extract [23], the hexadecanoic acid could possibly target the K-ras at this surface pocket.

The other ligands presented another interesting position. The pheophorbide epimers docked strongly into a depression on the surface of the K-ras that is also the surface for interaction with the SOS protein [35] The residues involved that formed the binding pocket for the epimers and 1hexadecene were Arg73, Thr74, Gly75, Glu76, and Gly77. The strong binding affinity between the protein and the epimers was the result of the hydrogen bonding between the ligands and Arg73 and Gly75.

For another docking experiment we used two of the PDB structures, 4LUC and 4LYF, reported by Ostrem et al. (2013) since it presented two different ligands, a sulphonamide and a vinyl sulphonamide that caused changes in the switch II region to accommodate the ligand [28]. The researchers focused on finding inhibitors that could bind to mutated GDP bound K-ras and change its structure such that it would not be able to exchange the GDP molecule for the GTP to be activated.

The authors showed that their test ligands targeted the switch II region that falls in the loop region between the central $\beta$-sheet, $\alpha 2$ and $\alpha 3$ helices of the ras. The pocket had been earlier reported by Taveras et al. [36]. We used the same binding pocket lined with residues Val7, Val9, Gly10, Ala11, Thr58, Ala59, Gly60, Gln61, Glu62, Glu63, Arg68, Tyr71, and Met72. Our results revealed that the pheophorbide epimers had the strongest affinity. However, the docked poses presented an entirely different picture.

In case of the structure PDB ID: 4LUC, pheophorbide a could insert its side chain $\left(-\mathrm{CH}_{2}-\mathrm{CH}_{2}-\mathrm{COOH}\right)$ into the pocket (Figure 2). The same side chain of pheophorbide $\mathrm{a}^{\prime}$ did not penetrate much. Pyropheophorbide a occupied position lined by residues Phe90, Glu91, His94, His95, Gln129, Asp132, Leu133, and Ser136. Being bulky molecules they could not penetrate inside the pocket, however could make a hydrogen bond with Glu63, His95 and Tyr96 that contributed to the binding affinity of -7.0 for pheophorbide a, 6.8 for pheophorbide $\mathrm{a}^{\prime}$ and $-7.3 \mathrm{Kcal} / \mathrm{mol}$ for pyropheophorbide a. Hexadecanoic acid and hexadecene could easily slide into the pocket and make polar contact with Arg68. This was perhaps due to the long hydrophobic chain despite the low

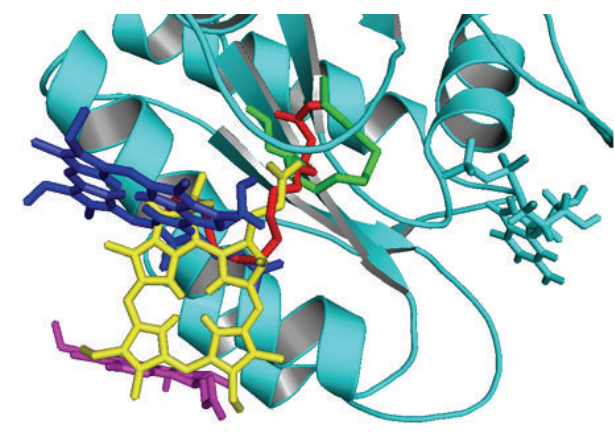

FIGURE 2: Docked poses of the flagelliforme constituents to the binding pocket of K-ras (PDB ID 4LUC). The ligands are hexadecanoic acid (red), pheophorbide a (yellow), phephorbide a' (blue pyropheophorbide a, and magenta), and 1-hexadecene (green)).

binding affinity. The pheophorbide a and a' showed that they occupied the binding region that included residues 61-64 and 68 on K-ras with pheophorbide a interacting with Glu63. As already pointed out [35] these residue are involved in the contact surface between the Ras-SOS proteins. Hence if pheophorbide is able to bind to these residues, it could prevent the interaction of the two proteins in a pronounced manner.

The other K-ras structure (PDB ID: 4LYF) has a vinylsulphonamide covalently bound to the K-ras in the same pocket area. The authors indicated that when this compound binds to the pocket, it modifies the switch II position and completely disorders switch I [25].

Our docking experiment given in Figure 3 on this expanded pocket allowed the 1-hexadecene and hexadecanoic acid to go deep into the pocket and occupy the same binding pocket as the inhibitor and formed hydrophobic interactions inside their binding pocket. As had been pointed out by the authors, the disorderliness of the switch regions caused by the ligands could lead to SOS protein not being able to bind efficiently to the ras protein, leading to inhibition of the exchange of nucleotides and ultimate inactivity of the protein [25]. However pheophorbides targeted the binding region comprised of Lys5, Arg73, Thr74, Gly75, Glu76, Val103, Lys104, Asp105, and Ser106 which according to BoriackSjodin et al. (1998) is part of the site of interaction between ras-SOS [35]. Pheophorbide a exhibited the highest affinity of $-6.7 \mathrm{Kcal} / \mathrm{mol}$, while pyropheophorbide a molecule with binding affinity $6.5 \mathrm{Kcal} / \mathrm{mol}$ formed a hydrogen bond with Arg73. Because of their big size and span of the ring, we can hypothise that these compounds cover the area of interaction between K-ras and SOS and, hence, may be important direct inhibitors of the K-ras at the ras-SOS interacting region.

Taking these results together, we can say that K-ras could be a good target for the pheophorbides of the flagelliforme. They have two hotspots on the protein. One is the region of surface contact between the SOS protein and the Kras and the other could be the switch two region. We carried out experiments in both the activated state and inactivated state and in both states. Our results showed that pheophorbides naturally targeted the SOS interacting residues preferable in the activated (4DST) and inactivated 


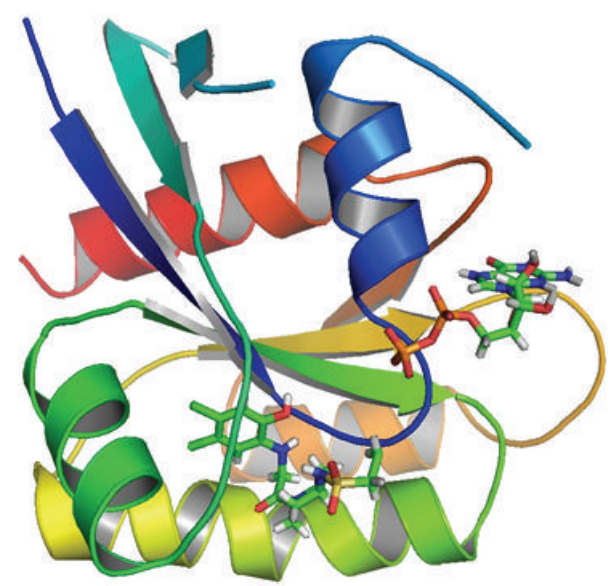

(a)

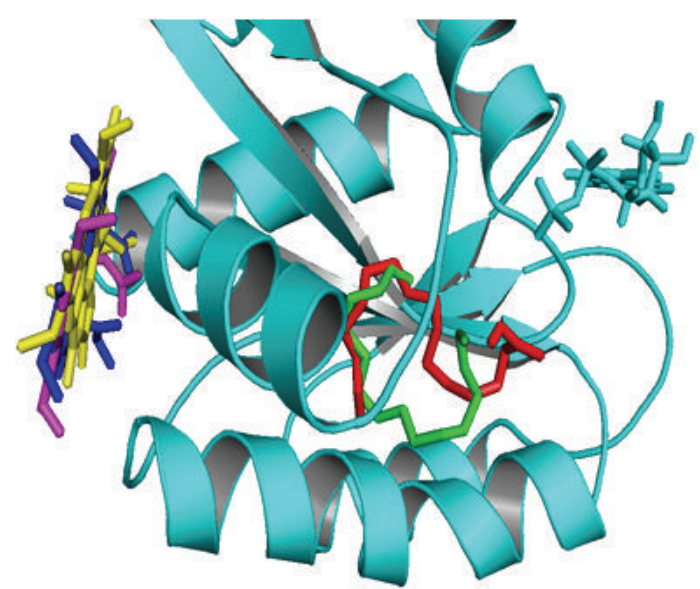

(b)

FIgURE 3: Docked poses of the flagelliforme constituents to the binding pocket. K-ras PDB ID 4LYF. (a) Control ligand vinylsulphonamide docked in the switch II region. (b) Test ligands pheophorbide a (yellow), pheophorbide a' (blue), and pyropheophorbide a (magenta) bound at the SOS-interaction site while 1-hexadecene (green) and hexadecanoic acid (red) are bound in the switch II region.

state (4LUC, 4LYF). The hexadecanoic acid and 1-hexadecene looked for hydrophobic pockets that it could slide into and their preferable hotspot was switch II.

\section{Conclusion}

Hence, we can conclude that T. flagelliforme constituents could target several allosteric sites on the K-ras. Since this protein is the most important signaling molecule in cancer cells inhibiting this protein would induce apoptosis of lung cancer cells. Combining our results with experimental evidence from Lai et al. $(2008,2010)$ where whole extracts are more potent than individual constituents, we can hypothesize that K-ras could have been the probable target of pheophorbides and other constituents. Pheophorbides bind to the SOS binding spot on K-ras and could possibly prevent a strong interaction between the nucleotide exchange protein SOS and $\mathrm{K}$-ras, while 1-hexadecane and hexadecanoic acid bind to switch II region of the K-ras. Both events combined would eventually inhibit the growth signals in the cancerous cells. Further studies are required to conclusively indicate K-ras at the target for the pheophorbides and other constituents.

\section{Conflict of Interests}

The authors declare that there is no conflict of interests regarding the publication of this paper.

\section{References}

[1] Z. A. Omar, Z. M. Ali, and S. I. Tamin Nor, Eds., Malaysian Cancer Statistics-Data and Figure Penisular Malysia, National Cancer Registry Ministry of Health Malaysia, 2006.

[2] National Cancer Society Malaysia, http://cancer.org.my/learnabout-cancer/about-cancer/.

[3] G. C. C. Lim, "Overview of cancer in Malaysia," Japanese Journal of Clinical Oncology, vol. 32, pp. S37-S42, 2002.
[4] S. Mohan, S. I. Abdelwahab, S.-C. Cheah et al., "Apoptosis effect of girinimbine isolated from Murraya koenigii on lung cancer cells in vitro," Evidence-based Complementary and Alternative Medicine, vol. 2013, Article ID 689865, 12 pages, 2013.

[5] C.-K. Liam, M. I. A. Wahid, P. Rajadurai, Y.-K. Cheah, and T. S.-Y. Ng, "Epidermal growth factor receptor mutations in lung adenocarcinoma in Malaysian patients," Journal of Thoracic Oncology, vol. 8, no. 6, pp. 766-772, 2013.

[6] The American Cancer Society, "Learn about cancer," http://www .cancer.org/cancer/lungcancer-non-smallcell/detailedguide/nonsmall-cell-lung-cancer-what-is-non-small-cell-lung-cancer.

[7] A. Fernández-Medarde and E. Santos, "Ras in cancer and developmental diseases," Genes and Cancer, vol. 2, no. 3, pp. 344-358, 2011.

[8] P. M. K. Westcott and M. D. To, "The genetics and biology of KRAS in lung cancer," Chinese Journal of Cancer, vol. 32, no. 2, pp. 63-70, 2013.

[9] M. V. Milburn, L. Tong, A. M. Devos et al., "Molecular switch for signal transduction: structural differences between active and inactive forms of protooncogenic ras proteins," Science, vol. 247, no. 4945, pp. 939-945, 1990.

[10] D. A. Eberhard, B. E. Johnson, L. C. Amler et al., "Mutations in the epidermal growth factor receptor and in KRAS are predictive and prognostic indicators in patients with nonsmall-cell lung cancer treated with chemotherapy alone and in combination with erlotinib," Journal of Clinical Oncology, vol. 23, no. 25, pp. 5900-5909, 2005.

[11] A. T. Brünger, M. V. Milburn, L. Tong et al., "Crystal structure of an active form of RAS protein, a complex of a GTP analog and the HRAS p21 catalytic domain," Proceedings of the National Academy of Sciences of the United States of America, vol. 87, no. 12, pp. 4849-4853, 1990.

[12] E. Massarelli, M. Varella-Garcia, X. Tang et al., "KRAS mutation is an important predictor of resistance to therapy with epidermal growth factor receptor tyrosine kinase inhibitors in nonsmall cell lung cancer," Clinical Cancer Research, vol. 13, no. 10, pp. 2890-2896, 2007.

[13] Y.-S. Chang, K.-T. Yeh, N. C. Hsu, S.-H. Lin, T.-J. Chang, and J.-G. Chang, "Detection of N-, H-, and KRAS codons 12, 13, and 
61 mutations with universal RAS primer multiplex PCR and N-, $\mathrm{H}$-, and KRAS-specific primer extension," Clinical Biochemistry, vol. 43, no. 3, pp. 296-301, 2010.

[14] M.-J. Yang, C.-K. Hsu, H.-J. Chang et al., “The KRAS mutation is highly correlated with EGFR alterations in patients with nonsmall cell lung cancer," Fooyin Journal of Health Sciences, vol. 1, no. 2, pp. 65-71, 2009.

[15] G. J. Riely, J. Marks, and W. Pao, "KRAS mutations in non-small cell lung cancer," Proceedings of the American Thoracic Society, vol. 6, no. 2, pp. 201-205, 2009.

[16] S. Jančík, J. Drábek, D. Radzioch, and M. Hajdúch, "Clinical relevance of KRAS in human cancers," Journal of Biomedicine and Biotechnology, vol. 2010, Article ID 150960, 13 pages, 2010.

[17] R. Dienstmann, S. De Dosso, E. Felip, and J. Tabernero, "Drug development to overcome resistance to EGFR inhibitors in lung and colorectal cancer," Molecular Oncology, vol. 6, no. 1, pp. 1526, 2012.

[18] J. C. Soria, T. S. Mok, F. Cappuzzo, and P. A. Jänne, "EGFRmutated oncogene-addicted non-small cell lung cancer: current trends and future prospects," Cancer Treatment Reviews, vol. 38, no. 5, pp. 416-430, 2012.

[19] P. Ulivi, D. Calistri, W. Zoli, and D. Amadori, "Predictive molecular markers for EGFR-TKI in non-small cell lung cancer patients: new insights and critical aspects," Journal of Nucleic Acids Investigation, vol. 1, no. 1, pp. 47-54, 2010.

[20] G. M. Stella, M. Luisetti, S. Inghilleri et al., "Targeting EGFR in non-small-cell lung cancer: lessons, experiences, strategies," Respiratory Medicine, vol. 106, no. 2, pp. 173-183, 2012.

[21] H.-F. Hsu, K.-H. Huang, K.-J. Lu et al., “Typhonium blumei extract inhibits proliferation of human lung adenocarcinoma A549 cells via induction of cell cycle arrest and apoptosis," Journal of Ethnopharmacology, vol. 135, no. 2, pp. 492-500, 2011.

[22] C. S. Lai, R. H. M. H. Mas, N. K. Nair, S. M. Mansor, and V. Navaratnam, "Chemical constituents and in vitro anticancer activity of Typhonium flagelliforme (Araceae)," Journal of Ethnopharmacology, vol. 127, no. 2, pp. 486-494, 2010.

[23] C.-S. Lai, R. H. M. H. Mas, N. K. Nair, M. I. A. Majid, S. M. Mansor, and V. Navaratnam, "Typhonium flagelliforme inhibits cancer cell growth in vitro and induces apoptosis: an evaluation by the bioactivity guided approach," Journal of Ethnopharmacology, vol. 118, no. 1, pp. 14-20, 2008.

[24] T. Maurer, L. S. Garrenton, A. Oh et al., "Small-molecule ligands bind to a distinct pocket in Ras and inhibit SOSmediated nucleotide exchange activity," Proceedings of the National Academy of Sciences of the United States of America, vol. 109, no. 14, pp. 5299-5304, 2012.

[25] B. J. Grant, S. Lukman, H. J. Hocker et al., "Novel allosteric sites on ras for lead generation," PLoS ONE, vol. 6, no. 10, Article ID e25711, 2011.

[26] F. Shima, Y. Yoshikawa, M. Ye et al., "In silico discovery of small-molecule Ras inhibitors that display antitumor activity by blocking the Ras-effector interaction," Proceedings of the National Academy of Sciences of the United States of America, vol. 110, no. 20, pp. 8182-8187, 2013.

[27] O. Trott and A. J. Olson, "Software news and update AutoDock Vina: improving the speed and accuracy of docking with a new scoring function, efficient optimization, and multithreading," Journal of Computational Chemistry, vol. 31, no. 2, pp. 455-461, 2010.

[28] J. M. Ostrem, U. Peters, M. L. Sos, J. A. Wells, and K. M. Shokat, "K-Ras(G12C) inhibitors allosterically control GTP affinity and effector interactions," Nature, vol. 503, no. 7477, pp. 548-551, 2013.

[29] G. M. Morris, D. S. Goodsell, R. S. Halliday et al., "Automated docking using a Lamarckian genetic algorithm and an empirical binding free energy function," Journal of Computational Chemistry, vol. 19, no. 14, pp. 1639-1662, 1998.

[30] G. Morris and R. Huey, "AutoDock4 and AutoDockTools4: automated docking with selective receptor flexibility," Journal of Computational Chemistry, vol. 30, pp. 2785-2791, 2009.

[31] R. Huey, G. M. Morris, and S. Forli, Using AutoDock 4 and Vina with AutoDockTools: A Tutorial, 2011.

[32] D. Seeliger and B. L. de Groot, "Ligand docking and binding site analysis with PyMOL and Autodock/Vina," Journal of Computer-Aided Molecular Design, vol. 24, no. 5, pp. 417-422, 2010.

[33] ACD/ChemSketch, Version 12, Advanced Chemistry Development, ACD/ChemSketch, Toronto, Canada, 2014, http://www .acdlabs.com/.

[34] S. Mohan, A. B. Abdul, S. I. Abdelwahab et al., "Typhonium flagelliforme induces apoptosis in CEMss cells via activation of caspase-9, PARP cleavage and cytochrome c release: its activation coupled with G0/G1 phase cell cycle arrest," Journal of Ethnopharmacology, vol. 131, no. 3, pp. 592-600, 2010.

[35] P. A. Boriack-Sjodin, S. M. Margarit, D. Bar-Sagi, and J. Kuriyan, "The structural basis of the activation of Ras by Sos," Nature, vol. 394, no. 6691, pp. 337-343, 1998.

[36] A. G. Taveras, S. W. Remiszewski, R. J. Doll et al., "Ras oncoprotein inhibitors: the discovery of potent, ras nucleotide exchange inhibitors and the structural determination of a drugprotein complex," Bioorganic and Medicinal Chemistry, vol. 5, no. 1, pp. 125-133, 1997. 

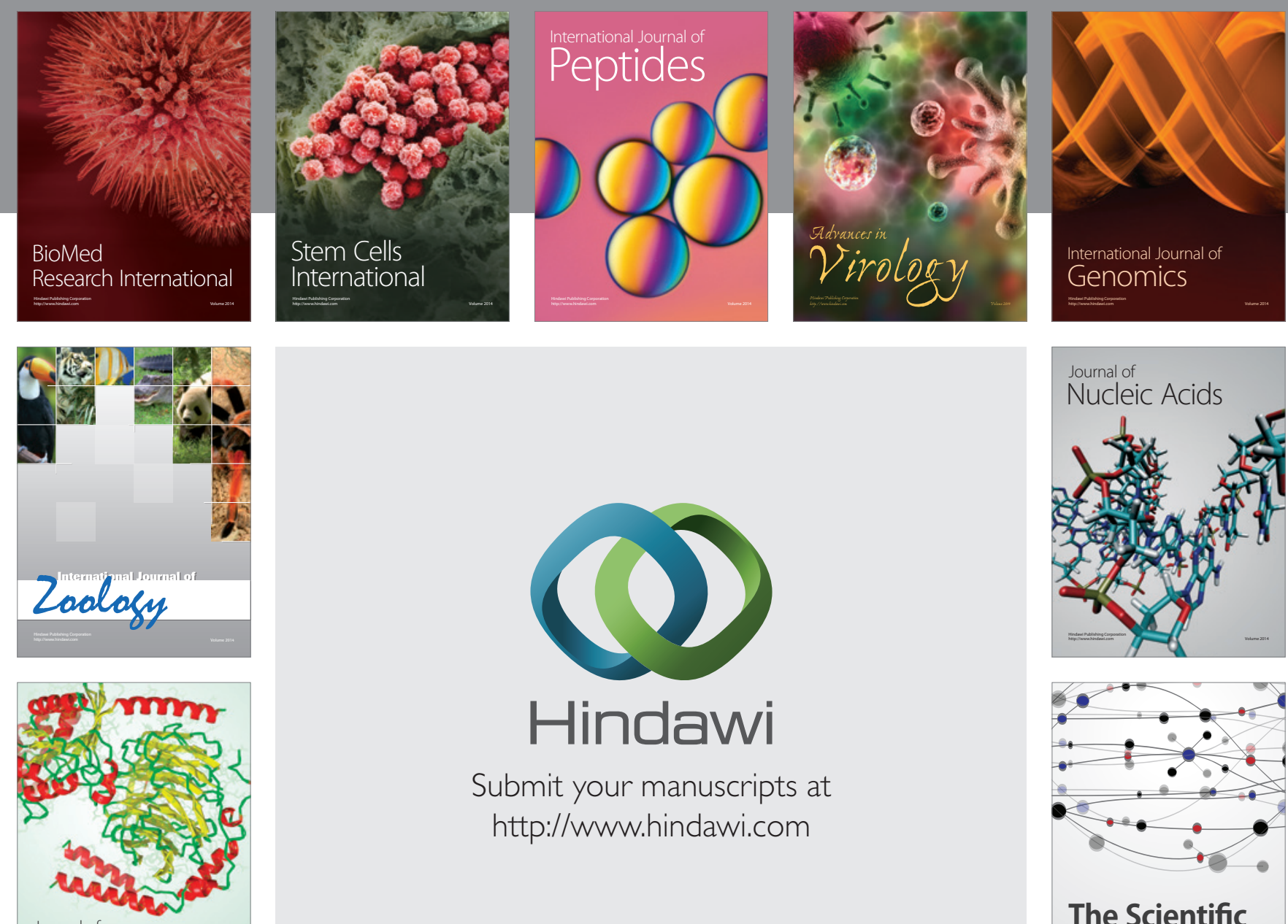

Submit your manuscripts at

http://www.hindawi.com

Journal of
Signal Transduction
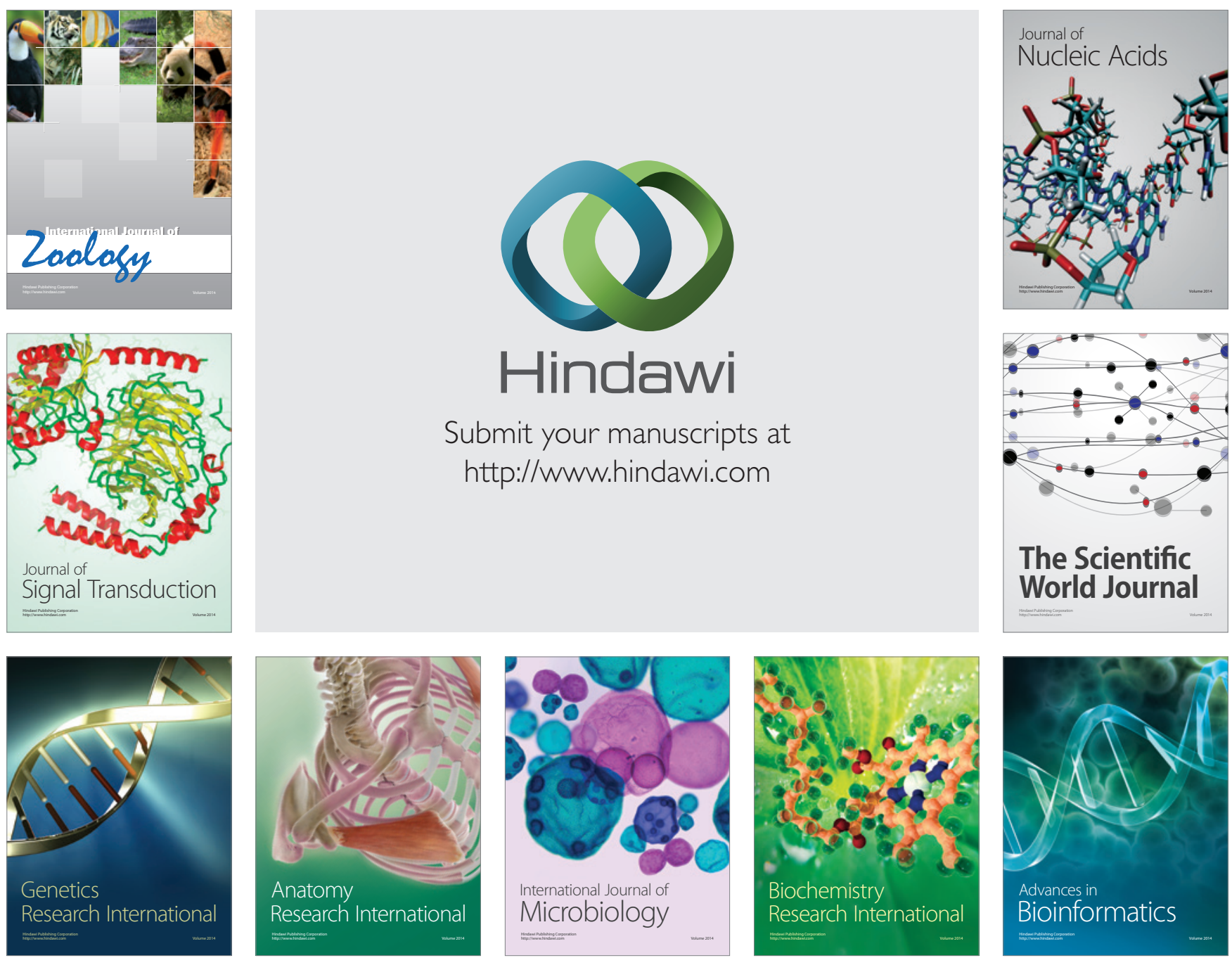

The Scientific World Journal
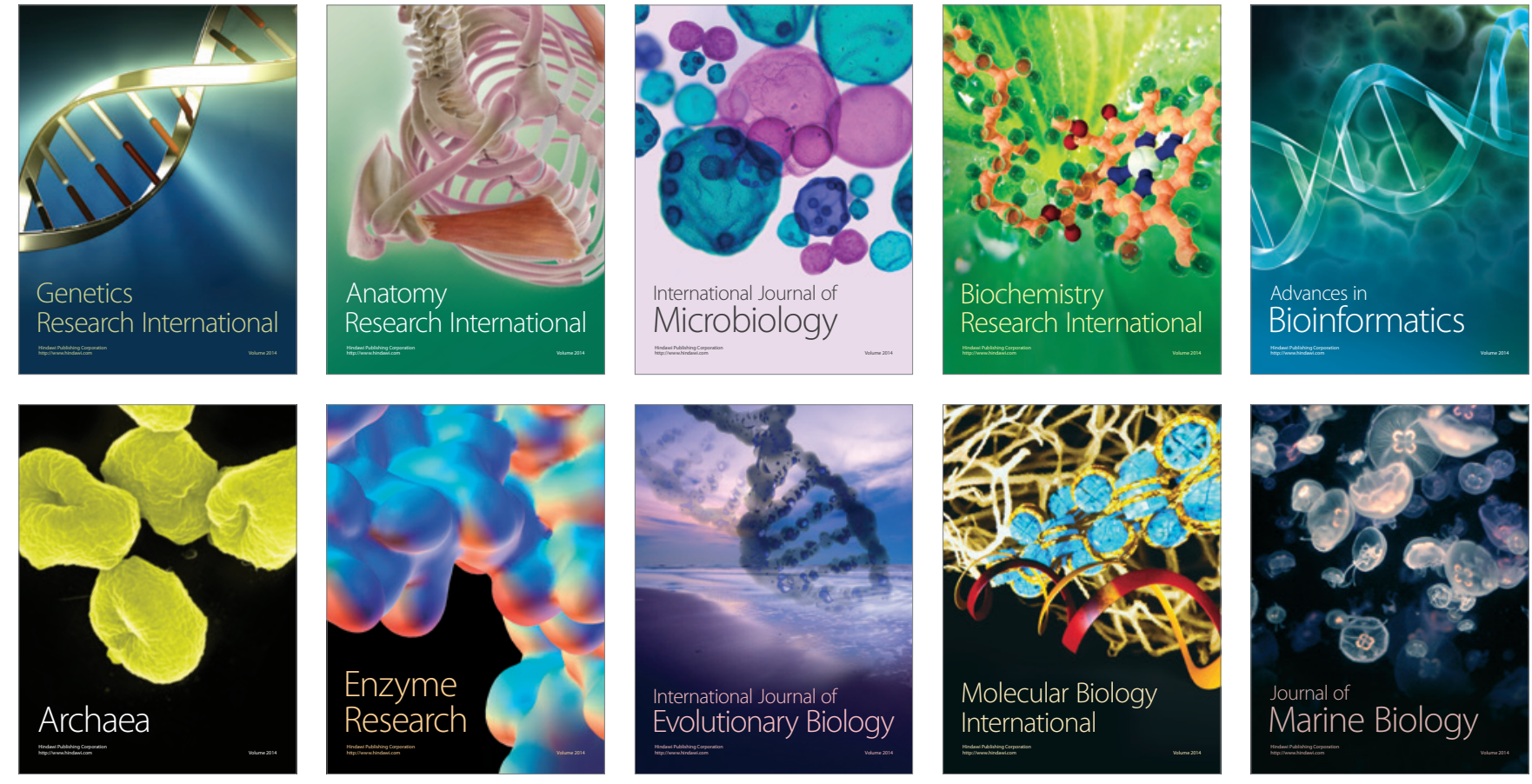